\title{
Focal adhesion kinase promotes progression and predicts poor clinical outcomes in patients with osteosarcoma
}

\author{
HUA-JIE GU and BIN ZHOU
}

Department of Orthopedics, People's Hospital of Yuyao, Yuyao, Zhejiang 315400, P.R. China

Received July 5, 2017; Accepted January 10, 2018

DOI: $10.3892 / \mathrm{ol} .2018 .8152$

\begin{abstract}
Osteosarcoma(OS) is a fatal form of musculoskeletal tumor that commonly leads to pulmonary metastatic disease. Traditional therapies such as surgery and chemotherapy are not effective treatment modalities in certain patients with OS; therefore, identifying the molecular mechanism of OS is imperative for the development of novel therapeutics. Previous studies have reported that focal adhesion kinase (FAK) is associated with numerous types of human malignancies. Therefore, in order to investigate the biological function of FAK in OS, the present study examined the expression levels of FAK in OS cell lines, OS tissues and paired normal tissue specimens by reverse transcription-quantitative polymerase chain reaction (RT-qPCR). FAK expression in vitro was blocked using small interfering RNA (siRNA) to observe the invasion, proliferation and apoptosis trends of OS cells. Phosphoinositide-dependent kinase-1 (PDK1), AKT and BRAF protein levels were also evaluated by western blotting to analyze the effects of FAK depletion on the AKT and mitogen-activated protein kinase (MAPK) signaling pathways. A significantly reduced level of FAK mRNA was identified in paired normal tissues compared with OS tissues and cell lines. The invasive capability and proliferative potential of OS cells were suppressed due to the transient in vitro transfection of FAK siRNA. It was also demonstrated that decreased FAK expression facilitated the apoptosis of OS cells, as demonstrated by flow cytometric and western blotting analyses. Decreased FAK expression resulted in the downregulation of phosphorylated (p)-AKT, p-PDK1 and p-BRAF protein levels. Higher FAK expression levels are positively associated with clinicopathological characteristics of advanced Enneking stages $(\mathrm{P}<0.001)$ and recurrence
\end{abstract}

Correspondence to: Dr Bin Zhou, Department of Orthopedics, People's Hospital of Yuyao, 800 Chengdong Road, Yuyao, Zhejiang 315400, P.R. China

E-mail: zhou_bin_cn@126.com

Abbreviations: OS, osteosarcoma; FAK, focal adhesion kinase; siRNA, small interfering RNA

Key words: osteosarcoma, focal adhesion kinase, migration, proliferation, prognosis
$(\mathrm{P}=0.041)$ in patients with OS. Collectively, these data demonstrated that FAK is an important diagnostic biomarker for OS, and FAK siRNA therapy may be a potentially promising approach for the treatment of OS.

\section{Introduction}

Osteosarcoma (OS) is the most commonly occurring type of primary malignant bone tumor; it primarily occurs in children and adolescents, with a current estimated incidence rate of $5.4 / 1,000,000$ worldwide $(1,2)$. Currently, the treatment of OS relies on the application of comprehensive treatments, including chemotherapy and surgery. The 5-year survival rate of OS has improved over the last 30 years since 1980, reaching $\sim 70 \%$ in the USA (3). However, there is still a considerable amount of patients with OS who have exhibited clinical insensitivity to the presently available chemotherapies, and these patients typically have a poor prognosis (4). Therefore, investigating novel strategies, such as translational research, is necessary to help broaden the understanding of OS and determine novel approaches for therapeutic modalities (5).

Focal adhesion kinase (FAK) is a $\sim 125-\mathrm{kDa}$ protein that is recruited as a participant in focal adhesion dynamics between cells, and serves a role in cellular motility and survival. FAK also serves a role in the protein-protein-interaction adaptor at the sites of cell attachment to the extracellular matrix (ECM), contributing to focal-adhesion 'scaffolding' and transmitting adhesion-dependent signals into the cell interior (6). A previous study has reported that FAK is involved in the regulation of the turnover of adhesion sites, which are vital in the control of cell migration (7).

In the context of cancer, a considerable amount of evidence has demonstrated increased FAK expression in tumor cells, including in cancers of the colon, rectum, oral epithelium and ovary $(8,9)$. Additionally, the phosphorylation of FAK at specific sites, for example, phosphorylation of FAK at Y397, has been demonstrated to be associated with numerous tumor types (10).

Although the inhibition of FAK function may be a promising anti-cancer approach, it remains obscure as to what the most effective modality would be. Due to the fact that FAK is a non-receptor protein-tyrosine kinase implicated in signaling pathways that affect cell migration, proliferation and apoptosis, and potentially serves a role in oncogenic transformation that lead to increased kinase activity, potential intervention routes have included the inhibition of kinase activity and the 
disruption of vital protein-protein interactions (6). The underlying mechanisms that can explain the increased expression of FAK in tumor cells have not been completely elucidated; however, the amplification of the FAK gene has been reported in a number of cancer cell lines $(6-9,11)$. The aim of the present study was to explore the role of FAK in the carcinogenesis and development of OS. Firstly, the expression levels of FAK in human OS tissues and cell lines were compared with normal adjacent tissues. Secondly, the expression of FAK was downregulated by transfecting the MG-63 cell line with FAK siRNA. in vitro and in vivo functional studies, including the Cell Counting Kit-8 (CCK-8) assay, Transwell invasion assay and flow cytometric analysis were conducted to measure the proliferation, migration and apoptosis of the cells, in order to examine the functional effects of FAK depletion in OS. Thirdly, western blotting analysis was conducted to evaluate the expression pattern of phosphorylated (p)-AKT, p-BRAF and p-PDK1 proteins to test their effects on the AKT and MAPK signaling pathways. It is well-established that the AKT and MAPK signaling pathways are involved in the biological activities of human cancer types, including cell survival, proliferation, differentiation and tumor growth $(12,13)$. FAK expression was determined using immunohistochemistry, and the association between FAK expression and clinicopathological characteristics was measured in the present study.

The data of the present study indicated that FAK expression is significantly upregulated in OS cell lines and tissues, compared with those in normal adjacent tissues. siRNA-induced depletion of FAK inhibited the proliferation and migration of the MG63 cell line. The increased expression of FAK is closely associated with advanced OS stage and higher rates of recurrence; therefore, FAK overexpression predicts an unfavorable clinical outcome in patients with OS.

\section{Materials and methods}

OS cell culture and cell lines. Two OS cell lines, MG-63 and U2-OS, were purchased from the American Type Culture Collection (ATCC; Manassas, VA, USA). OS cells were maintained in a humidified atmosphere containing $5 \% \mathrm{CO}_{2}$ at $37^{\circ} \mathrm{C}$. Cell lines were cultured in Dulbecco's modified Eagle's medium (DMEM; Sigma-Aldrich; Merck KGaA, Darmstadt, Germany) and supplemented with $10 \%$ heat-inactivated fetal bovine serum (FBS), $100 \mathrm{U} / \mathrm{ml}$ penicillin and $100 \mathrm{mg} / \mathrm{ml}$ streptomycin (Sigma-Aldrich; Merck KGaA), according to the established protocols of the ATCC.

Patient and specimen samples. Human OS samples and matched normal adjacent tissue samples were obtained from 80 patients, who were diagnosed with OS and were admitted for treatment at the Department of Orthopedics of the People's Hospital of Yuyao (Yuyao, China). The inclusion criteria for OS patients were as follows: i) Patients had readily available and clear clinical data; ii) patients had not previously received any antitumor treatments, such as radiotherapy or chemotherapy; and iii) none of the patients presented with any genetic disorders or other diseases of the bone. A total of 80 OS tissue samples and 80 paired normal adjacent tissue samples were obtained from orthopedic surgery. The collected tissues were divided into two groups, each group had
40 OS tissue samples and 40 paired normal tissue samples, for different experimental purposes: i) One was frozen immediately following surgery and was stored at $-80^{\circ} \mathrm{C}$ until RNA isolation; ii) the other group was fixed in $10 \%$ buffered formaldehyde solution and then was embedded in paraffin and sectioned, according to the standard protocol of the Society for Applied Immunohistochemistry (14). The study design complied with the regulation of the Helsinki Declaration, and the present study was approved by the Ethics Committee of the People's Hospital of Yuyao (Yuyao, China). All patients agreed to participate in the study with informed written consent.

Transient siRNA transfection. Functional analyses used siRNA duplexes specific for FAK to knockdown FAK expression, which were designed by and purchased from GenePharma Co., Ltd. (Shanghai, China). The transient transfections of siRNA were carried out using Lipofectamine ${ }^{\circledR} 2000$ (Invitrogen; Thermo Fisher Scientific, Inc., Waltham MA, USA). OS cells were plated at $\sim 80 \%$ confluence in DMEM containing $10 \%$ serum with no antibiotics. Transfections were performed within $<20 \mathrm{~h}$ after plating. FAK siRNA and Lipofectamine ${ }^{\circledR}$ 2000 were diluted with $100 \mu 1$ serum-free DMEM. After 5 min, the diluted FAK siRNA was mixed with diluted Lipofectamine 2000 (Invitrogen; Thermo Fisher Scientific, Inc.), and the mixture was incubated for $30 \mathrm{~min}$ at room temperature to ensure complex formation. FAK siRNA was transfected at the concentration of $50 \mathrm{nmol} / \mathrm{l}$. The knockdown effect was confirmed using reverse transcription-quantitative polymerase chain reaction (RT-qPCR). The FAK siRNA sequence was as follows: FAK siRNA forward, 5'-GCCTGG TGAAAGCTGTCATC-3' and reverse, 5'-GCTTCTGTGCCA TCTCAATC-3'. A scrambled siRNA that did not target FAK was used as control. The sequence of the scrambled siRNA was as follows: Sense, 5'-ACGCGUAACGCGGGAAUUUdT dT-3' and antisense, 5'-AAAUUCCCGCGUUACGCGUdT dT-3'.

RT-qPCR analysis. Total RNAs were extracted from OS tissues, OS cell lines and paired normal adjacent tissues using TRIzol $^{\circledR}$ (Thermo Fisher Scientific, Inc.) and a RecoverAll ${ }^{\mathrm{TM}}$ Total Nucleic Acid Isolation kit (Ambion; Thermo Fisher), according to the manufacturer's protocol. The experiment was performed as follows: RNAs were reverse transcribed using the SYBR Green PrimeScript ${ }^{\mathrm{TM}}$ RT-PCR kit (cat no. RR066A; Takara Biotechnology Co., Ltd., Dalian, China). The GAPDH primer sequence used in the study was as follows, sense 5'-CAGAAGACUGUGGAUGGCC-3', anti-sense 3'-GGC CAUCCACAGUCUUCUG-5'. RT-qPCR conditions were as follows: Denaturing at $95^{\circ} \mathrm{C}$ for $5 \mathrm{~min}$, prior to 40 cycles of amplification at $95^{\circ} \mathrm{C}$ for $30 \mathrm{sec}$, then $60^{\circ} \mathrm{C}$ for $30 \mathrm{sec}$ and $72^{\circ} \mathrm{C}$ for $30 \mathrm{sec}$. Each sample was assayed in triplicate to quantify the mean $2^{-\Delta \Delta \mathrm{Cq}}$ values, \pm standard deviation (SD) (15). FAK miRNA expression level was normalized to the relative quantities of GAPDH.

Western blot analysis. Proteins were quantified using the BCA protein assay kit (Pierce; Thermo Fisher Scientific Inc.). The following primary antibodies used in the present study were purchased from Abcam (Cambridge, MA, USA.); pan-AKT antibody (dilution 1:1,000; catalog no. ab8805); p-AKT3 (S472) 
+ AKT2 (S474) + AKT1 (S473) antibody (dilution 1:1,000, catalog no. ab192623); BRAF antibody (dilution 1:1,000; catalog no. ab33899); p-BRAF (T401) antibody (dilution 1:800; catalog no. ab68215); PDK1 antibody (dilution 1:1,000; catalog no. ab52893); p-PDK1 (S241) antibody (dilution 1:800; catalog no. ab32800); BAX antibody (dilution 1:1,000; catalog no. ab32503), Bcl-2 antibody (dilution 1:1,000; catalog no. ab59348). A $\beta$-actin antibody (dilution 1:1,000; catalog no. A2066) purchased from Sigma-Aldrich (Merck KGaA) was used as the endogenous control. Total proteins were extracted by lysing cultured cells using RIPA buffer, and cell debris were removed by centrifuging the lysates at $15,000 \mathrm{x}$ g for $15 \mathrm{~min}$ at $4^{\circ} \mathrm{C}$. The protein extracts (50 $\mu \mathrm{g}$ per lane) were separated and fractionated using $12 \%$ SDS-PAGE and were then transferred to PVDF membranes (Merck Millipore). The PVDF membranes were blocked with PBS buffer supplemented with $5 \%$ bovine serum albumin (Sigma-Aldrich; Merck KGaA) overnight at $4{ }^{\circ} \mathrm{C}$, and were then incubated with the primary antibodies at room temperature for $2 \mathrm{~h}$, followed by incubation with horseradish peroxidase-conjugated goat anti-rabbit IgG antibody (1:10,000; catalog no. ab205718; Abcam) at room temperature for another $2 \mathrm{~h}$. Following washing with tris-buffered saline with $0.1 \%$ Tween-20, the bands of interest were visualized using ECL (EMD Millipore, Billerica, MA, USA).

Immunohistochemistry. Formalin-fixed paraffin-embedded (FFPE) whole tissue specimens were sequentially cut into $4-\mu$ m-thick sections. Following de-paraffinization and rehydration, the sections were cut into $4 \mu \mathrm{m}$ thick sections, and were dewaxed in xylene, followed by heating in a microwave at the temperature of $60^{\circ} \mathrm{C}$ for $30 \mathrm{~min}$ in EDTA buffer (pH 9.0) for antigen retrieval. FFPE sections were treated with $0.3 \% \mathrm{H}_{2} \mathrm{O}_{2}$, and then incubated with $10 \%$ normal goat serum (Sigma-Aldrich; Merck KGaA). Antigen retrieval was conducted using EDTA (pH 8.0) at $100^{\circ} \mathrm{C}$ for $25 \mathrm{~min}$. Sections were washed, and then incubated with the FAK antibody (dilution 1:60; catalog no. ab40794; Abcam) overnight at $4^{\circ} \mathrm{C}$. Afterwards, FFPE sections were incubated at $37^{\circ} \mathrm{C}$ for $40 \mathrm{~min}$, prior to washing and incubation with the secondary antibody at a dilution of 1:200 (cat. no. 9720; Fuzhou Maixin Biotech Co., Ltd., Fuzhou, China) at room temperature for $60 \mathrm{~min}$. FFPE sections were washed and incubated with diaminobenzidine tetrachloride for $15 \mathrm{~min}$, and were counterstained with hematoxylin for $20 \mathrm{~min}$ at room temperature. Meanwhile, negative controls (NC) were prepared and incubated with PBS, without the FAK antibody. FAK immunohistochemistry was independently evaluated by two experienced pathologists from the People's Hospital of Yuyao (Yuyao, China), and were blinded to patient characteristics. The percentage of positively stained cells in the representative fields, were calculated and the images were photographed using a Leica DM IRB microscope (magnification, x200 and x400; Leica Micosystems $\mathrm{GmbH}$, Wetzlar, Germany). FAK levels in tissue samples (OS tissues and paired normal adjacent tissues) were measured using a semi-quantitative scoring method $(16,17)$, based on the intensity and percentage of overall staining. The staining intensities of FAK were grouped according to the following criteria: $<5 \%$ FAK-positive tumor cells was classified as negative staining; 6-25\% FAK-positive tumor cells was classified as weak staining; $25-50 \%$ FAK-positive tumor cells was classified as moderate staining; and $>50 \%$ FAK-positive tumor cells was classified as strong staining.

Cell apoptosis assay using flow cytometric analysis. Given that the expression of FAK mRNA was lower in MG-63 cells than that in U2-OS cells, the depletion of FAK mRNA was greater, and so the MG- 63 cell line was used in subsequent knockdown assays. MG-63 cells were plated on 6-well plates at a density of $2 \times 10^{5}$ cells per well, were maintained in the medium overnight. Afterwards, cells were trypsinized, collected, centrifuged, and washed three times with cold PBS, and were harvested and re-suspended in $250 \mu \mathrm{l} 1 \mathrm{X}$ binding buffer at a density of $1 \times 10^{6}$ cells $/ \mathrm{ml}$. Cells were transferred to a $5 \mathrm{ml}$ culture tube, and then were stained with $5 \mu \mathrm{l}$ Annexin $\mathrm{V}(1 \mathrm{mg} / \mathrm{ml}$; Beckman Coulter, Inc., Brea, CA, USA) at room temperature for $15 \mathrm{~min}$. Propidium iodide (PI; $1 \mathrm{mg} / \mathrm{ml}$ ) was subsequently used for cell staining for $5 \mathrm{~min}$ at room temperautre. Cells were vortexed gently and were incubated at room temperature in the dark for $15 \mathrm{~min}$. A total of $400 \mu \mathrm{l}$ of $1 \mathrm{X}$ Binding buffer was added into each tube, prior to assessment of the cell lines with a flow cytometer. Each experiment was conducted three times, independently.

Cell proliferation assay. The effect of FAK siRNA on the proliferation of OS cells was measured using CCK-8 (cat. no. 1166; R\&D Systems, Inc., Minneapolis, MN, USA). Cells were seeded into the 96 -well plates (10,000 cells per well). CCK8 was used according to the manufacturer's protocol, and $24 \mathrm{~h}$ after the transfection of FAK siRNA into the MG-63 cell line, CCK-8 was added into each well and then was incubated at $37^{\circ} \mathrm{C}$ for $1.5 \mathrm{~h}$. Optical density was measured at $450 \mathrm{~nm}$ using a microplate spectrophotometer (Tecan Group Ltd., Zurich, Switzerland).

Cell invasion assay. The invasive ability of OS cells was determined using 24-well Transwell plates (Corning Life Sciences, Tewksbury, MA, USA) pre-coated with Matrigel basement membrane matrix, with a concentration of $1 \mathrm{mg} / \mathrm{ml}$. A total of $2 \times 10^{4}$ cells were seeded into each well in the upper chamber with $0.1 \%$ serum. In the lower chamber, $0.6 \mathrm{ml}$ of medium containing $10 \%$ FBS was added to stimulate invasion. Transwell plates were incubated overnight at $37^{\circ} \mathrm{C}$ in an atmosphere of $5 \% \mathrm{CO}_{2}$. Non-invading cells were removed from the top chamber, while invading cells in the bottom chamber were fixed with $4 \%$ paraformaldehyde, stained with $0.1 \%$ crystal violet. The images were photographed using Olympus CKX53 Inverted Microscope (x200 magnification). Data collected were based on three independently conducted experiments.

Statistical analysis. Data are expressed as the mean \pm SD and were analyzed using the SPSS statistical software package version 21.0 (IBM Corp., Armonk, NY, USA). One-way analysis of variance followed Scheffe's test, or the Student's t-test was used to analyze the differences in the expression levels of FAK mRNA. Pearson's $\chi^{2}$ tests and Fisher's exact test were used to analyze the association of FAK expression with clinicopathological characteristics. $\mathrm{P}<0.05$ was considered to indicate a statistical significant difference. 

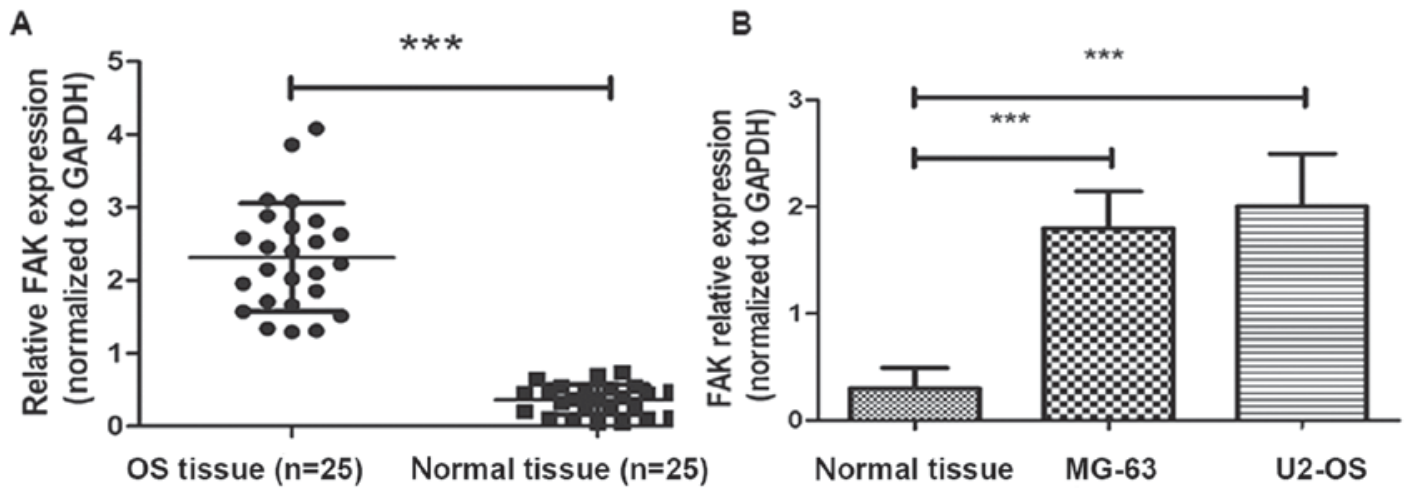

Figure 1. RT-qPCR analysis of FAK expression levels (normalized to GAPDH). (A) FAK mRNA levels in OS tissue samples were significantly enhanced compared with those in the normal adjacent tissue samples $(\mathrm{P}<0.001)$. (B) FAK mRNA expression levels were significantly upregulated in MG-63 and U2-OS cell lines compared with those in normal adjacent tissues $(\mathrm{P}<0.001)$. Data are presented as the mean \pm standard deviation. ${ }^{* * *} \mathrm{P}<0.01$. FAK, focal adhesion kinase; OS, osteosarcoma.
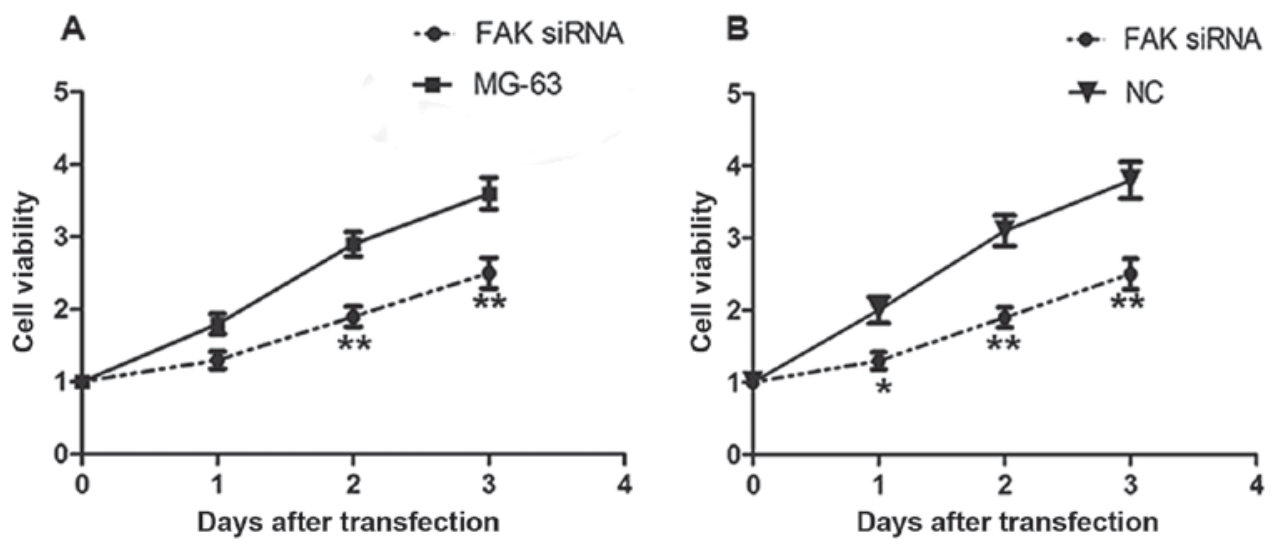

Figure 2. Comparison of cell viability among non-transfected MG-63 cells and MG-63 cells transfected with non-silencing scramble siRNA or with FAK siRNA. (A) A CCK-8 assay demonstrated that the proliferation of OS cells was suppressed 48 and $72 \mathrm{~h}$ following the transfection of FAK siRNA (P<0.01 vs. control) compared with that of the untransfected MG-63 cells; (B) A CCK-8 assay demonstrated that the proliferation of OS cells was inhibited at 48 and $72 \mathrm{~h}$ following the transfection of FAK siRNA ( $\mathrm{P}<0.01 \mathrm{vs.} \mathrm{control)} \mathrm{compared} \mathrm{with} \mathrm{cell} \mathrm{line} \mathrm{treated} \mathrm{with} \mathrm{the} \mathrm{scramble} \mathrm{control} \mathrm{siRNA.} \mathrm{Data} \mathrm{are} \mathrm{presented} \mathrm{in} \mathrm{the} \mathrm{form}$ of the mean \pm standard deviation. Data were collected from independently conducted experiments. ${ }^{*} \mathrm{P}<0.05$ and ${ }^{* *} \mathrm{P}<0.01 \mathrm{vs}$. MG- 63 or NC group. FAK, focal adhesion kinase; NC, negative control; OS, osteosarcoma; CCK-8, Cell Counting Kit-8.

\section{Results}

FAK expression level is upregulated in human OS tissues and cell lines compared with that in normal adjacent tissues. To analyze the expression levels of FAK mRNA, RT-qPCR analysis was conducted in the OS tissue samples, paired normal adjacent samples, MG-63 and U2-OS cell lines. GAPDH was used as a reference gene. As shown in Fig. 1A, FAK mRNA expression was significantly lower in paired normal tissues compared with the levels in OS tissues and cell lines $(\mathrm{P}<0.001)$.

siRNA-induced depletion of FAK and suppresses the proliferation of MG-63 cells. To investigate the biological role of FAK on the proliferation of OS, FAK downregulation was conducted by transfecting MG-63 cells with FAK siRNA. Following transfection, CCK- 8 was used to measure the effect of FAK downregulation on the proliferation of MG-63 cells (Fig. 2). As illustrated in Fig. 2, the downregulation of FAK significantly suppressed the growth of MG-63 cells after 48 and $72 \mathrm{~h}$ of transfection, compared with that of the NC group and untrasfected MG-63 cell. The results demonstrated that the siRNA-mediated inhibition of FAK inhibited the proliferation of OS cells.

siRNA-mediated knockdown of FAK inhibites the invasion of OS cells. To evaluate the role of FAK in the invasive potential of MG-63 OS cells transfected with FAK siRNA, Transwell assays were performed. As presented in Fig. 3, the invasive potential of OS cells was suppressed after the downregulation of FAK (MG-63 cell line transfected with FAK siRNA), compared with those of non-transfected MG-63 cells and the scrambled siRNA transfection group. The invaded cell numbers in the FAK siRNA group were the smallest of the three groups analyzed.

siRNA-mediated downregulation of FAK induces the apoptosis of OS cells and enhances the expression of apoptotic proteins. To investigate the mechanism behind the association of FAK with the regulation of OS cell growth, cell apoptosis was measured by flow cytometry. As shown in Fig. 4, the percentage of apoptotic OS cells was significantly lower in 
A

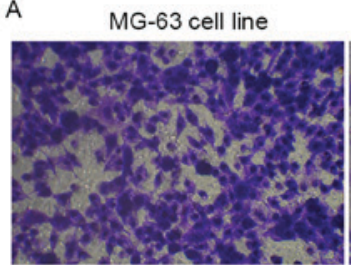

FAK siRNA transfected

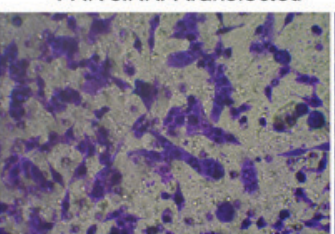

NC

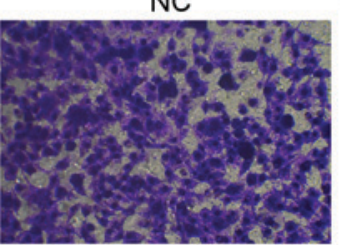

B

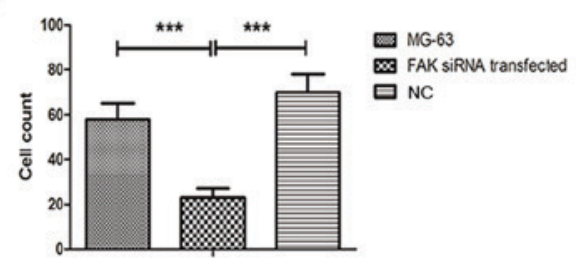

Figure 3. siRNA-induced knockdown of FAK suppresses the invasion of MG-63 cells. (A) Representative images of a Transwell invasion assay demonstrated that invasion of MG-63 cells was inhibited following transfection with FAK siRNA (magnification, $\mathrm{x} 200$ ). (B) Invasive cell numbers in FAK siRNA transfected MG-63 cells were significantly reduced, compared with those in the NC group and untransfected MG- 63 cells $(\mathrm{P}<0.001)$. All experiments were performed in triplicate, independently. ${ }^{* * *} \mathrm{P}<0.001$ (normal vs. FAK depletion; NC vs. FAK depletion). FAK, focal adhesion kinase; NC, negative control.

A.

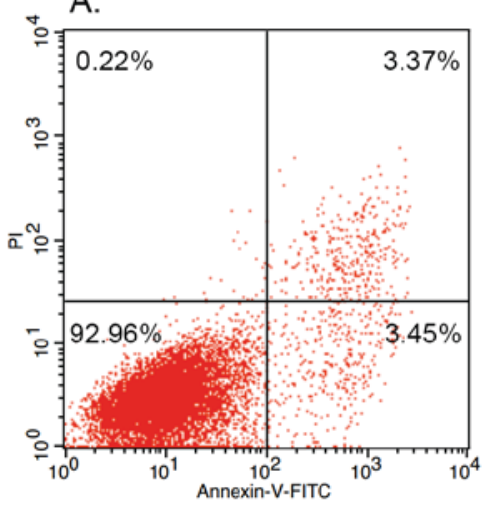

B.

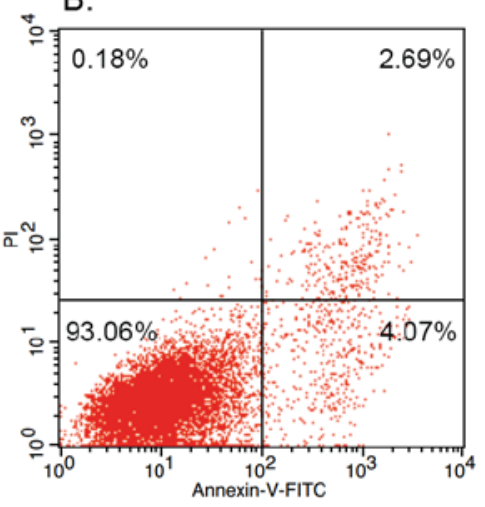

C.

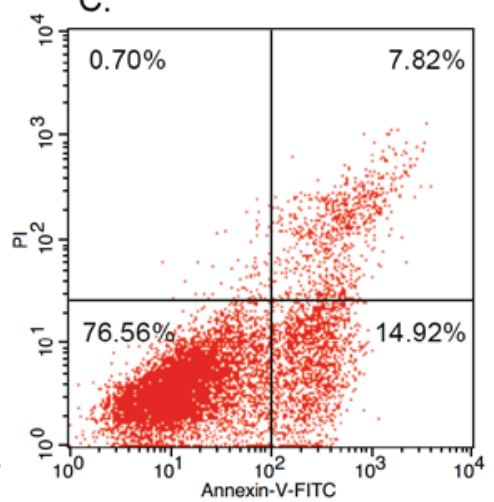

Figure 4. Early apoptosis analysis was performed by flow cytometry after staining with Annexin V-FITC/PI. (A) Early apoptosis analysis of non-transfected MG-63 cells; (B) early apoptosis analysis of MG-63 cells transfected with negative control siRNA; (C) early apoptosis analysis of MG-63 cells transfected with FAK siRNA. FAK, focal adhesion kinase; FITC, fluorescein isothiocyanate; PI, propidium iodide; UL, upper left; UR, upper right; LL, lower left; LR, lower right.

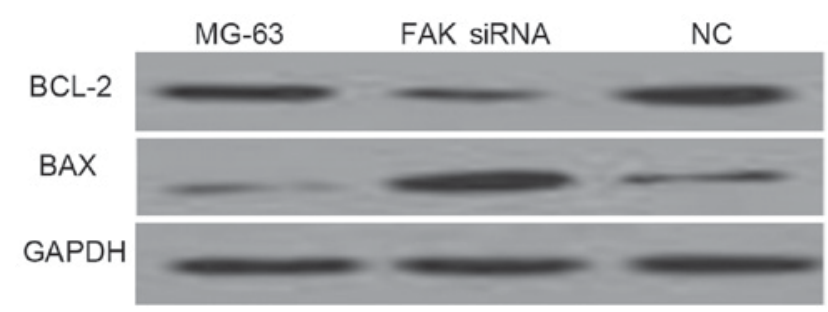

Figure 5. FAK depletion induces apoptosis in OS cells. For OS cells transfected with FAK siRNA, the levels of Bax (a pro-apoptotic regulator) were upregulated whereas levels of Bcl-2, (an anti-apoptosis regulator), were downregulated. GAPDH was used as an internal control. FAK, focal adhesion kinase; $\mathrm{NC}$, negative control.

the group of untransfected MG-63 cells (3.45\%; Fig. 4A) and the NC siRNA group (4.07\%; Fig. 4B) when compared with the siRNA-induced FAK knockdown (14.92\%) group (Fig. 4C).

Western blot analysis of Bax and Bcl-2 antibodies demonstrated that FAK depletion induces apoptosis. As shown in Fig. 5, the expression of Bax was increased and the expression of Bcl-2 was decreased in MG-63 cells transfected with FAK siRNA, when compared with the negative control (NC) group and untransfected MG-63 cells. Consistent with the results obtained by flow cytometry, western blot analysis of Bax and Bcl-2 demonstrated that down-regulation of FAK induces apoptosis in OS cells.

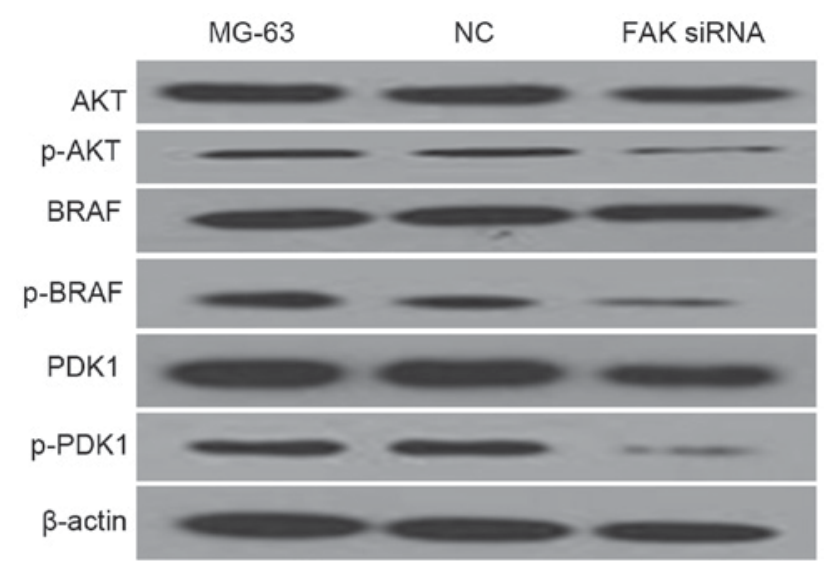

Figure 6. Western blot analysis of proteins involved in the AKT and MAPK signaling pathways. FAK, focal adhesion kinase; NC, negative control.

FAK down-regulation is associated with the deactivation of AKT and MAPK pathways. PDK1 is a component of the AKT pathway, while BRAF is a component of the MAPK pathway. In the western blot analysis of the present study, AKT, p-AKT, PDK1 p-PDK1, BRAF, p-BRAF antibodies were used to measure the protein levels of their corresponding genes in FAK-depleted MG-63 cells. $\beta$-actin was used as endogenous control. As shown in Fig. 6, p-AKT, p-PDK1 and p-BRAF protein levels in the FAK-depleted MG-63 cell line were significantly lower than those in other examined groups. 

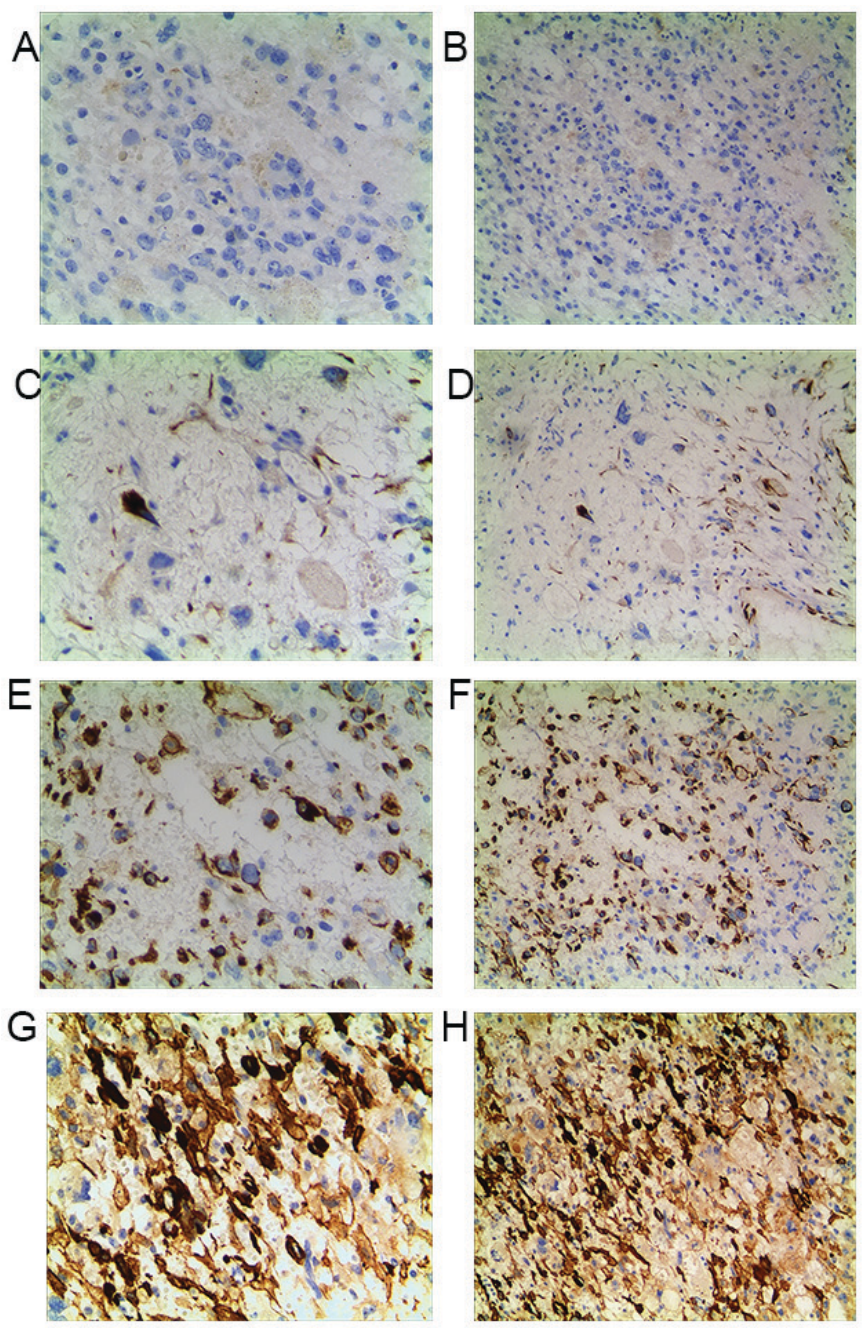

Figure 7. Immunohistochemical staining of FAK in OS tissue and normal adjacent tissue samples. Representative images of FAK expression are shown for paired normal tissues (A and B) (negative); (C and D) OS tissue (weak staining); (E and F) OS tissue section (intermediate staining); and ( $\mathrm{G}$ and $\mathrm{H}$ ) OS sections (strong staining). Representative images are shown at x400 (left) and x200 (right) magnification using light microscopy. FAK, focal adhesion kinase; OS, osteosarcoma.

The data demonstrated that the AKT and MAPK pathways are involved in the down-regulation of FAK.

Elevated FAK expression suggests an unfavorable prognosis in patients with $O S$. To further determine the clinicopathological significance of FAK in OS, we performed IHC analysis of FAK in tissues collected from 80 cases of OS. Representative IHC images of FAK expression are shown in Fig. 7.

The associations between FAK expression level and the corresponding clinicopathological characteristics of patients with OS were calculated using Pearson's $\chi^{2}$ test. Associations between FAK expression level and the clinicopathological features of 80 patients with OS patients are summarized in Table I, based on the IHC results of FAK protein expression (Fig. 7). The Enneking surgical staging system was consulted when measuring the grade of OS (18). Stage I represents low grade, stage II tumors are high grade and stage III represents tumors with distant metastasis. The present study identified that clinically advanced Enneking stages $(\mathrm{P}<0.001)$ and
Table I. Expression levels of FAK and their associations with the clinicopathological features of the 80 patients with osteosarcoma included in the present study.

\begin{tabular}{lcccc}
\hline & & \multicolumn{2}{c}{ FAK expression } & \\
\cline { 3 - 4 } $\begin{array}{l}\text { Clinicopathological } \\
\text { characteristics }\end{array}$ & Total, $\mathrm{n}$ & High, $\mathrm{n}$ & Low, n & P-value \\
\hline Sex & & & & 0.739 \\
$\quad$ Male & 42 & 17 & 25 & \\
Female & 38 & 14 & 24 & \\
Age, years & & & & 0.183 \\
$\quad<18$ & 47 & 20 & 27 & \\
$\geq 18$ & 33 & 19 & 14 & \\
Recurrence & & & & $0.041^{\mathrm{a}}$ \\
$\quad$ Yes & 51 & 35 & 16 & \\
No & 29 & 10 & 19 & \\
Enneking stage & & & & $<0.001^{\mathrm{a}}$ \\
II & 31 & 12 & 19 & \\
III & 49 & 35 & 14 & \\
\hline
\end{tabular}

FAK, focal adhesion kinase; $n$, number of patients. ${ }^{a} \mathrm{P}<0.05$ FAK high expression vs. low expression; Pearson's $\chi^{2}$ tests and Fisher's exact test.

recurrence $(\mathrm{P}=0.041)$ in patients with $\mathrm{OS}$ were associated with higher FAK expression levels.

\section{Discussion}

OS ranks as the third most common type of cancer in adolescents, following lymphomas and brain tumors (11). Despite advancements in the diagnosis and treatment of OS in recent years, its clinical prognosis has not considerably improved. Furthermore, due to the high mortality and morbidity rates, rapid progression and poor prognosis of OS caused by the migration of OS cells, OS continues to pose a severe threat to the health of patients $(3,6)$. Metastasis remains the principal cause for the mortality of OS patients (19). Therefore, determining an accurate biomarker for OS by extensive investigation may help direct the development of novel therapies. The metastasis of tumors is a complex biological and pathological process, in which the studies by Liotta et al $(20,21)$ summarized that three steps are included: i) Adhesion; ii) degradation; iii) migration and invasion.

FAK is a non-receptor protein tyrosine kinase that is widely expressed in the cytoplasm. The phosphorylation of FAK can mediate numerous downstream signaling pathways, incurring various biological processes within the cells. Phosphorylation of FAK mediates signaling pathways and is widely involved in various biological processes within cells, and contributes to tumor progression, including invasion, metastasis and angiogenesis (22-25). The study by Ilić et al (26) demonstrated that FAK is involved in the turnover of focal adhesion contacts during cell migration in FAK-deficient mice. It was also reported that the association of FAK with signaling proteins, such as PI3-kinase and paxillin, enables FAK to function in a network of integrin-stimulated signaling pathways, giving rise 
to the activation of ERK and JNK/MAPK (27). The present study demonstrated the down-regulation of p-AKT, p-PDK1 and p-BRAF proteins in the MG-63 cell line following transfection with FAK siRNA. These results demonstrated that decreased expression of FAK results in the deactivation of the AKT and MAPK pathways.

Considerable evidence has indicated that the pro-apoptotic regulator Bax serves a role in accelerating programmed cell death by antagonizing or binding to the apoptosis repressor Bcl-2 (28). Bcl-2 is involved in variety of cell systems, and regulates cell death by regulating the permeability of the mitochondrial membrane. Consequently, changes in the expression patterns of Bax and Bcl-2 are indicative of cell apoptosis (29). In the present study, the data revealed that the expression of the pro-apoptotic protein Bax was increased in the FAK-depleted cell line, while the expression level of the anti-apoptotic protein Bcl-2 was decreased. Furthermore, flow cytometric analysis demonstrated that the down-regulation of FAK in the MG-63 cell line resulted in an increased percentage of early apoptotic cells. These observations demonstrated that depleted FAK expression stimulated apoptosis in OS cells.

Clinicopathological parameters, including Enneking stage, metastasis and recurrence rates, are key indicators for the progression and deterioration of OS $(18,30)$. The in vitro and in vivo data of the present study are in agreement with the results of a previous study, which reported that increased expression of FAK was associated with tumor progression and a worse prognosis (31). The present study postulates that overexpressed FAK promotes malignancy and eventually results in poorer clinical outcomes in patients with OS. Consequently, OS patients with a higher expression level of FAK are anticipated to have a shorter overall survival time.

The limitations of the present study is that the data acquired were based on in vitro experiments, and the present study explored the biological role FAK in OS cells by depleting FAK expression, while the effect of FAK upregulation on OS cells was not addressed. The comparison of FAK mRNA expression was conducted using RT-qPCR; and the FAK protein levels in untransfected MG-63 cells and cells transfected with FAK siRNA or scrambled control were not assessed by western blotting.

To conclude, the present study comprehensively demonstrated that FAK plays a role in the proliferation, migration and apoptosis of OS cells through the AKT and MAPK pathways, and that enhanced FAK expression was positively associated with aggressive clinicopathological characteristics of patients with OS. These observations may provide basis for novel therapeutic strategies targeting FAK in the treatment of OS.

\section{Acknowledgements}

The authors thank Professor Juan Huang (Department of Oncology, People's Hospital of Yuyao, Yuyao, China) for revising the manuscript.

\section{Funding}

The present study was supported by the National Natural Science Foundation of China (grant no. 53048352) and the People's Hospital of Yuyao.

\section{Availability of data and materials}

All data generated or analyzed in the present study to establish the conclusion are available and included in this article.

\section{Authors' contributions}

BZ obtained the funding and designed the study. HJG performed the experiments, collected, analyzed and interpreted the data. BZ wrote the manuscript. All authors have read and approved the manuscript.

\section{Ethics approval and consent to participate}

The study design complied with the regulation of the Helsinki Declaration and the present study was approved by the Ethics Committee of the People's Hospital of Yuyao (Yuyao, China). All patients provided written informed consent prior to their inclusion in the study.

\section{Consent for publication}

All patients consented to the publication of their data.

\section{Competing interests}

The authors declare that they have no competing interests.

\section{References}

1. Siclari VA and Qin L: Targeting the osteosarcoma cancer stem cell. J Orthop Surg Res 5: 78, 2010.

2. Isakoff MS, Bielack SS, Meltzer P and Gorlick R: Osteosarcoma: Current treatment and a collaborative pathway to success. J Clin Oncol 33: 3029-3035, 2015.

3. Allison DC, Carney SC, Ahlmann ER, Hendifar A, Chawla S, Fedenko A, Angeles C and Menendez LR: A meta-analysis of osteosarcoma outcomes in the modern medical era. Sarcoma 2012: 704872, 2012.

4. Pakos EE and Ioannidis JP: The association of P-glycoprotein with response to chemotherapy and clinical outcome in patients with osteosarcoma. A meta-analysis. Cancer 98: 581-589, 2003.

5. Golubovskaya MV: Focal adhesion kinase as a cancer therapy target. Anticancer Agents Med Chem 10: 735-741, 2010.

6. McLean GW, Carragher NO, Avizienyte E, Evans J, Brunton VG and Frame MC: The role of focal-adhesion kinase in cancer-a new therapeutic opportunity. Nat Rev Cancer 5: 505-515, 2005.

7. Weiner TM, Liu ET, Craven RJ and Cance WG: Expression of focal adhesion kinase gene and invasive cancer. Lancet 342: 1024-1025, 1993.

8. Kornberg LJ: Focal adhesion kinase and its potential involvement in tumor invasion and metastasis. Head Neck 20: 745-752, 1998.

9. Lark AL, Livasy CA, Calvo B, Caskey L, Moore DT, Yang X and Cance WG: Overexpression of focal adhesion kinase in primary colorectal carcinomas and colorectal liver metastases: Immunohistochemistry and real-time PCR analy. Clin Cancer Res 9: 215-222, 2003.

10. Grisaru-Granovsky S, Salah Z, Maoz M, Pruss D, Beller U and Bar-Shavit R: Differential expression of protease activated receptor 1 (Par1) and pY397FAK in benign and malignant human ovarian tissue samples. Int J Cancer 113: 372-378, 2005.

11. Wittig JC, Bickels J, Priebat D, Jelinek J, Kellar-Graney K, Shmookler B and Malawer MM: Osteosarcoma: A multidisciplinary approach to diagnosis and treatment. Am Fam Physician 65: 1123-1132, 2002.

12. Vivanco I and Sawyers CL: The phosphatidylinositol 3-kinase-AKT pathway in human cancer. Nat Rev Cancer 2: 489-501, 2002.

13. Fang JY and Richardson BC: The MAPK signalling pathways and colorectal cancer. Lancet Oncol 6: 322-327, 2005. 
14. Sakr RA, Barbashina V, Morrogh M, Chandarlapaty S, Andrade VP, Arroyo CD, Olvera N and King TA: Protocol for PTEN expression by immunohistochemistry in formalin-fixed paraffin-embedded human breast carcinoma. Appl Immunohistochem Mol Morphol 18: 371-374, 2010.

15. Livak KJ and Schmittgen TD: Analysis of relative gene expression data using real-time quantitative PCR and the 2(-Delta Delta $\mathrm{C}(\mathrm{T})$ ) method. Methods 25: 402-408, 2001

16. Lassmann S, Bauer M, Soong R, Schreglmann J, Tabiti K, Nährig J, Rüger R, Höfler H and Werner M: Quantification of CK20 gene and protein expression in colorectal cancer by RT-PCR and immunohistochemistry reveals inter- and intratumour heterogeneity. J Pathol 198: 198-206, 2002.

17. Bhargava R, Beriwal S and Dabbs DJ: Mammaglobin vs GCDFP-15: An immunohistologic validation survey for sensitivity and specificity. Am J Clin Pathol 127: 103-113, 2007.

18. Jawad MU and Scully SP: In brief: Classifications in brief: Enneking classification: Benign and malignant tumors of the musculoskeletal system. Clin Orthop Relat Res 468: 2000-2002, 2010.

19. Liu X, Zeng B, Ma J and Wan C: Comparative proteomic analysis of osteosarcoma cell and human primary cultured osteoblastic cell. Cancer Invest 27: 345-352, 2009.

20. Liotta LA, Steeg PS and Stetler-Stevenson WG: Cancer metastasis and angiogenesis: An imbalance of positive and negative regulation. Cell 64: 327-336, 1991.

21. Liotta LA and Kohn EC: The microenvironment of the tumour-host interface. Nature 411: 375-379, 2001.

22. Mak M, Reinhart-King CA and Erickson D: Microfabricated physical spatial gradients for investigating cell migration and invasion dynamics. PLoS One 6: e20825, 2001.

23. Zachary I: Focal adhesion kinase. Int J Biochem Cell Biol 29 929-934, 1997

24. Beviglia L, Golubovskaya V, Xu L, Yang X, Craven RJ and Cance WG: Focal adhesion kinase $\mathrm{N}$-terminus in breast carcinoma cells induces rounding, detachment and apoptosis. Biochem J 373: 201-210, 2003.
25. Sakurai S, Sonoda Y, Koguchi E, Shinoura N, Hamada H and Kasahara T: Mutated focal adhesion kinase induces apoptosis in a human glioma cell line, T98G. Biochem Biophys Res Commun 293: 174-181, 2002.

26. Ilić D, Furuta Y, Kanazawa S, Takeda N, Sobue K, Nakatsuji N, Nomura S, Fujimoto J, Okada M and Yamamoto T: Reduced cell motility and enhanced focal adhesion contact formation in cells from FAK-deficient mice. Nature 377: 539-544, 1995.

27. Schlaepfer DD, Hauck CR and Sieg DJ: Signaling through focal adhesion kinase. Prog Biophys Mol Biol 71: 435-478, 1999.

28. De Angelis PM, Stokke T, Thorstensen L, Lothe RA and Clausen OP: Apoptosis and expression of Bax, Bcl-x, and Bcl-2 apoptotic regulatory proteins in colorectal carcinomas, and association with p53 genotype/phenotype. Mol Pathol 51: 254-261, 1998.

29. Kluck RM, Bossy-Wetzel E, Green DR and Newmeyer DD: The release of cytochrome $\mathrm{c}$ from mitochondria: A primary site for Bcl-2 regulation of apoptosis. Science 275: 1132-1136, 1997.

30. Parkes SE, Parke S, Mangham DC, Grimer RJ, Davies P and Morland BJ: Fifty years of paediatric malignant bone tumours in the West Midlands, UK, 1957-2006: Incidence, treatment and outcome. Paediatr Perinat Epidemiol 24: 470-478, 2010.

31. Miyazaki T, Kato H, Nakajima M, Sohda M, Fukai Y, Masuda N, Manda R, Fukuchi M, Tsukada K and Kuwano H: FAK overexpression is correlated with tumour invasiveness and lymph node metastasis in oesophageal squamous cell carcinoma. Br J Cancer 89: 140-145, 2003.

This work is licensed under a Creative Commons Attribution-NonCommercial-NoDerivatives 4.0 International (CC BY-NC-ND 4.0) License. 